ЦЕНТРАЛЬНОЕ АОРТАЛЬНОЕ ДАВЛЕНИЕ И АРТЕРИАЛЬНАЯ РИГИДНОСТЬ ПРИ СУТОЧНОМ МОНИТОРИРОВАНИИ У ЖЕНЩИН С ПОСТМЕНОПАУЗАЛЬНЫМ ОСТЕОПОРОЗОМ

(с) С.Ю. Царенок, В.В. Горбунов, Т.А. Аксенова

ФГБОУ ВО «Читинская государственная медицинская академия», Чита, Россия

ЦЕЛЬ: оценить показатели центрального аортального давления и артериальной ригидности при суточном мониторировании у женщин в постменопаузе в зависимости от наличия остеопороза.

МЕтоды: обследовано 79 постменопаузальных женщин в возрасте от 57 до 78 лет. Все пациентки были разделены на две группы: первая группа 36 человек - пациентки с установленным диагнозом остеопороз, вторая группа - 43 человека группа сравнения. Всем пациенткам проводилось суточное мониторирование центрального AД аппаратом BPLabv. 3.2 («Петр Телегин», Россия).

РЕЗУЛЬТАТЫ: у женщин с остеопорозом выявлено повышение среднесуточных показателей САД, ДАД и среднего давления в аорте. Установлено, что в данной группе пациентов увеличены основные показатели жесткости артерий: CPПВ, ASI, AASI, PPA. Выявлена прямая корреляционная связь между параметрами центрального давления, артериальной ригидности и наличием в анамнезе переломов, их количеством, а также показателями абсолютного десятилетнего риска остеопоротических переломов и перелома шейки бедра. У пациенток с остеопорозом чаще выявляются патологические профили систолического давления в аорте, преобладающим из которых был тип гипердиппер.

ЗАКЛЮчЕНИЕ: полученные результаты могут свидетельствовать о возможной взаимосвязи между сердечно-сосудистыми заболеваниями и остеопорозом.

КЛЮЧЕВЫЕ СЛОВА: Остеопороз; аортальное давление; артериальная ригидность

\title{
THE CENTRAL AORTIC BLOOD PRESSURE AND ARTERIAL STIFFNESS DURING THE DAILY MONITORING PROCEDURE IN POSTMENOPAUSAL WOMEN WITH OSTEOPOROSIS
}

(c) Svetlana Yu. Tsarenok, Vladimir V. Gorbunov, Tatiana A. Aksenova

Chita State Medical Academy, Chita, Russia

AIM: to evaluate the data of the central aortic pressure and arterial stiffness during the daily monitoring procedure in postmenopausal women with osteoporosis.

METHODS: 79 postmenopausal women (age from 57 to 78) were examined. All patients were divided into two groups: the first group consisted of 36 women with osteoporosis, the second group consisted of 43 women - control group. A daily monitoring of central aortic pressure and arterial stiffness were performed all women by the apparatus BPLab v.3.2.

RESULTS: increase of the mean daily of systolic, diastolic and mean aortic pressure was found out in women with osteoporosis. The main data of arterial stiffness (PWV, ASI, AASI, PPA) were higher in this group of women. The direct correlation between the data of central aortic blood pressure, arterial stiffness and presence of osteoporotic fractures and their number, as well as indicators of absolute ten-year risk of osteoporotic fractures and hip fracture was revealed. Pathological profiles of systolic pressure in aortae were more frequent in patients with osteoporosis. The type of hyperdispers was predominant.

CONCLUSIONS: the results obtained may indicate a possible relationship between cardiovascular diseases and osteoporosis.

KEYWORDS: Osteoporosis; aortic blood pressure; arterial stiffness

\section{ОБОСНОВАНИЕ}

В последнее время внимание исследователей направлено на изучение параметров центрального давления в аорте, измеряемого неинвазивным методом, а также сопряженных с ним показателей, отражающих артериальную жесткость. Такой интерес объясняется тем, что центральное аортальное давление и параметры сосудистой жесткости являются лучшими предикторами сердечно-сосудистых событий, чем артериальное давление, измеряемое на периферии [1]. Развитие сердечно-сосудистых заболеваний ассоциировано с повышением артериальной жесткости [2-4]. Этот интегральный показатель используется для анализа гемодинамических изменений, наблюдаемых при различных клинических состояниях. На артериальную жесткость оказывают влияние возраст, артериальная гипертензия, курение и т.д. [5,6]. При повышении ригидности артерий возрастает скорость пульсовой волны и отраженная волна возвращается в аорту во время систолы, что приводит к повышению пульсового давления и давления ауг- 
ментации. Эти показатели центрального давления могут использоваться для оценки сосудистой жесткости. Кроме того, центральное давление отражает кровоток в коронарных и мозговых артериях в аорте и является более значимым предиктором сердечно-сосудистых событий.

Проблемами позднего постменопаузального периода сердечно-сосудистые заболевания и остеопороз, приводящие к преждевременной смерти. Не проявляя себя клинически на ранних этапах развития, в последующем приводят к повышению заболеваемости, смертности и требуют значительных материальных затрат [7]. Обсуждаются общие патогенетические механизмы развития сердечно-сосудистых заболеваний и остеопороза. В свою очередь остеопороз рассматривается как один из факторов риска сердечно-сосудистых событий и компонент сердечно-сосудистого континуума [7]. Так, было продемонстрировано, что у женщин с низкой минеральной плотностью кости чаще развивается тяжелый коронарный атеросклероз, повышается риск инфарктов и инсультов $[8,9]$. Имеются исследования, посвященные артериальной ригидности у женщин с остеопорозом, где авторы изучали скорость пульсовой волны (СРПВ) и толщину комплекса интима-медиа [10], однако в доступной литературе мы не нашли данных о показателях центрального аортального давления и СРПВ при их суточном мониторировании у женщин с наличием постменопаузального остеопороза. В последние годы появилась возможность измерения показателей центрального давления и СРПВ в течение суток с использованием технологии Vasotens. Показано, что сфигмограмма, записанная на плечевой артерии, в качестве входного сигнала также позволяет воссоздать показатели центрального аортального давления посредством математической обработки. B программном обеспечении аппарата BPLab, который используется для этого исследования, расчет СРПВ проводится с использованием времени распространения отраженной от бифуркации аорты пульсовой волны таким образом, чтобы оно совпадало со СРПВ на каротидно-феморальном сегменте. Данный расчет позволяет сравнивать показатели артериальной ригидности, полученные посредством BPLab с измерениями на Sphigmocor. В литературе имеются данные о высокой сопоставимости этих методов [11].

\section{ЦЕЛЬ}

Оценить показатели центрального аортального давления и артериальной ригидности при суточном мониторировании у женщин в постменопаузе в зависимости от наличия остеопороза.

\section{МЕТОДЫ}

\section{Дизайн исследования}

Проведено наблюдательное одномоментное сплошное одноцентровое неконтролируемое исследование.

\section{Критерии соответствия}

Критериями включения в исследование были: женский пол, возраст старше 50 лет, наличие менопаузы. Критерии исключения: заболевания эндокринной системы, тяжелая сердечная недостаточность, дыхательная недостаточность, вторичный остеопороз.

\section{Условия проведения}

Исследование проведено на базе НУЗ «Дорожная клиническая больница на ст. Чита-2»

Продолжительность исследования

Исследование проведено в период с сентября 2011 по май 2012 гг.

\section{Описание медицинского вмешательства}

Для верификации остеопороза всем женщинам проводился сбор анамнеза с целью выявления факторов риска остеопороза и перенесенных низкоэнергетических переломов, рентгеновская остеоденситометрия в двух областях - поясничных позвонках и шейке бедра. Диагноз постменопаузального остеопороза выставлялся согласно Клиническим рекомендациям по профилактике и ведению больных с остеопорозом 2012 года [12]. Кроме того, всем пациенткам производился расчет абсолютного десятилетнего риска переломов - major osteoporotic (МО) - риск остеопоротических переломов любой локализации и hip fracture (HF) - риск перелома шейки бедра. Всем женщинам проводили суточное мониторирование артериального давления (СМАД). СМАД проводили до назначения антигипертензивных препаратов или через 2 суток после их отмены.

\section{Основной исход исследования}

Конечными точками исследования выступали показатели центрального аортального давления: систолическое давление в аорте (САДао), диастолическое давление в аорте (ДАДао), среднее давление в аорте (Ср АДао), пульсовое давление в аорте.

\section{Дополнительные исходы исследования}

Дополнительно анализировали параметры: абсолютный десятилетний риск остеопоротических переломов, наличие остеопоротических переломов, количество переломов в анамнезе, а также дополнительные параметры артериальной жесткости: индекс аугментации (Alхао), амплификация пульсового давления (РPA), - рассчитывается как отношение пульсового давления плечевой артерии к центральному пульсовому, скорость пульсовой волны (СРПВ), индекс ригидности артерий (ASI), амбулаторный индекс ригидности (AASI), индекс аугментации средний за сутки (АІхао ср.), индекс аугментации средний за сутки, приведенный к ЧСС (Alхао ср. к ЧСС), среднесуточное пульсовое АД (Пульсовое АД среднее). Определяли суточные профили центрального давления для САД и ДАД.

\section{Анализ в подгруппах}

Все женщины, участвующие в исследовании были разделены на две группы. Деление по группам проводили в зависимости от установленного диагноза остеопороза. Первую группу составили женщины в постменопаузе с установленным диагнозом остеопороза, 36 человек, вторая группа - 43 женщины явилась группой сравнения.

\section{Методы регистрации исходов}

Для выявления факторов риска остеопороза и остеопоротических переломов использовали анкету из онлайн программы FRAX и производили сбор анамнеза с целью установления количества остеопоротических 
переломов. Рентгеновская денситометрия проведена на аппарате Challenger, Франция. Оценка абсолютного десятилетнего риска остеопоротических переломов производили при помощи программы FRAX, размещенной на сайте www.shef.ac.uk/FRAX, русскоязычная версия, Российская модель на основании оценки клинических факторов и показателя минеральной плотности костной ткани шейки бедра. Суточное мониторирование центрального аортального давления и параметров артериальной жесткости выполнено на аппарате BPLab v. 3.2 («Петр Телегин», Россия).

\section{Этическая экспертиза}

Исследование одобрено локальным этическим комитетом при ГБОУ ВПО ЧГМА (протокол №20 от 22.03.2011г.).

\section{Статистический анализ}

Статистическую обработку проводили при помощи программного обеспечения Statistica 6.0 с использованием непараметрических критериев поскольку распределение признаков было ассиметричным: Вальда-Вольфовица, хи-квадрат. Корреляционный анализ выполнен с использованием ранговой корреляции Спирмена (для количественных величин) и гамма корреляции - для установления взаимосвязи между наличием в анамнезе низкоэнергетических переломов (учитывался как качественный признак) и параметрами центрального давления и артериальной ригидности. Достоверными считали отличия при $\mathrm{p}<0,05$.

\section{РЕЗУЛЬТАТЫ}

\section{Объекты (участники) исследования}

Первую группу составили пациентки с установленным диагнозом остеопороза - 36 женщин, вторая группа явилась группой сравнения - 43 женщины. Группы были сопоставимы по возрасту, весу и ИМТ. Абсолютный десятилетний риск остеопоротических переломов и перелома шейки бедра был статистически значимо выше в первой группе. Среднее количество переломов в первой группе

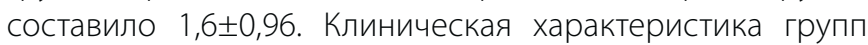
представлена в таблице 1.

У 79 женщин было проведено суточное мониторирование центрального давления и параметров артериальной ригидности, определялись суточные профили центрального давления исходя из степени ночного снижения систолического и диастолического давлений.

\section{Основные результаты исследования}

При анализе показателей центрального аортального давления, приведенных в таблице 2, у женщин с остеопорозом отмечается повышение среднесуточных показателей САД на 4,52\%, ДАД на 8,3\%, среднего АД в аорте на 5,24\%, давления амплификации в аорте (РPA) на 6,7\% по сравнению с контрольной группой (см. таб. 2).

Таблица 1. Клиническая характеристика пациентов

\begin{tabular}{|c|c|c|c|}
\hline Показатель & $\begin{array}{c}\text { Женщины с остеопорозом, } \\
\text { n=36 }\end{array}$ & $\begin{array}{c}\text { Группа сравнения, } \\
\text { n=43 }\end{array}$ & $P$ \\
\hline Возраст, лет & $69,4 \pm 9,1$ & $66,7 \pm 9,5$ & 0,3 \\
\hline POCT, CM & $159,4 \pm 6,3$ & $155,23 \pm 7,05$ & $<0,0001$ \\
\hline Bec, кг & $69,6 \pm 10,9$ & $68,5 \pm 13,6$ & 0,7 \\
\hline ИМТ & $27,1 \pm 3,89$ & $27,9 \pm 4,1$ & 0,46 \\
\hline Количество остеопоротических переломов в анамнезе & $1,6 \pm 0,96$ & $0,0 \pm 0$ & $<0,0001$ \\
\hline $\mathrm{MO}$ & $20,1 \pm 7,82$ & $8.5 \pm 4,59$ & $<0,0001$ \\
\hline $\mathrm{HF}$ & $7,98 \pm 7,27$ & $2,53 \pm 3,05$ & $<0,0001$ \\
\hline
\end{tabular}

Таблица 2. Параметры центрального аортального давления и артериальной ригидности при суточном мониторировании у женщин с остеопорозом

\begin{tabular}{|c|c|c|c|}
\hline Показатель & $\begin{array}{c}\text { Женщины с остеопорозом, } \\
\text { n=36 }\end{array}$ & $\begin{array}{c}\text { Группа сравнения, } \\
\mathrm{n}=43\end{array}$ & $P$ \\
\hline САД ао & $123,23 \pm 11,45$ & $117,66 \pm 10,05$ & 0,014 \\
\hline ДАД ао & $77,17 \pm 9,19$ & $70,73 \pm 9,98$ & 0,0008 \\
\hline Ср АД ао & $99,47 \pm 10,41$ & $94,26 \pm 9,47$ & 0,32 \\
\hline Пульсовое АД ао & $46,05 \pm 8,31$ & $46,04 \pm 9,43$ & 0,03 \\
\hline Alxao & $31,75 \pm 10,43$ & $34,9 \pm 13,28$ & 0,001 \\
\hline PPA & $118,7 \pm 4,41$ & $110,73 \pm 22,57$ & 0,09 \\
\hline $\mathrm{CP} \sqcap \mathrm{B}, \mathrm{M} / \mathrm{C}$ & $8,4 \pm 1,62$ & $8,24 \pm 0,9$ & 0,01 \\
\hline ASI, мM рт. Ст & $164,64 \pm 32,79$ & $162,06 \pm 32,53$ & 0,0002 \\
\hline Alxao cp.,\% & $1,64 \pm 18,06$ & $5,12 \pm 18,03$ & 0,000078 \\
\hline Alxao cp. к 4СC, \% & $-6,11 \pm 25,20$ & $-13,13 \pm 27,73$ & 0,0004 \\
\hline Пульсовое АД среднее & $52,87 \pm 10,05$ & $52,16 \pm 10,67$ & $<0,0001$ \\
\hline AASI, условные ед. & $0,54 \pm 0,23$ & $0,40 \pm 0,22$ & 0,0004 \\
\hline
\end{tabular}


Таблица 3. Корреляционные взаимосвязи между параметрами центрального давления, артериальной ригидности и наличием в анамнезе остеопоротических переломов, их количеством, риском переломов по FRAX

\begin{tabular}{|c|c|c|c|c|c|c|c|}
\hline Показатель & САДао & ДАДао & Ср АДао & $\begin{array}{l}\text { Alxao cp. } \\
\text { к ЧСС }\end{array}$ & ASI & AASI & Ср ПАД \\
\hline $\begin{array}{l}\text { Переломы в ана- } \\
\text { мнезе }\end{array}$ & $\begin{array}{c}\gamma=0,45, \\
p=0,00075\end{array}$ & $\begin{array}{c}\gamma=0,43 \\
p=0,0024\end{array}$ & $\begin{array}{c}\gamma=0,46, \\
p=0,0002\end{array}$ & $\begin{array}{c}\gamma=0,37 \\
p=0,0021\end{array}$ & НД & $\begin{array}{c}\gamma=0,37 \\
p=0,0021\end{array}$ & НД \\
\hline $\begin{array}{l}\text { Количество } \\
\text { переломов }\end{array}$ & $\begin{array}{c}r=0,33 \\
p=0,0016\end{array}$ & $\begin{array}{c}r=0,34 \\
p=0,002\end{array}$ & $\begin{array}{c}r=0,33 \\
p=0,0018\end{array}$ & $\begin{array}{c}r=0,33 \\
p=0,0016\end{array}$ & HA & $\begin{array}{c}r=0,36 \\
p=0,0012\end{array}$ & $\mathrm{HA}$ \\
\hline $\mathrm{MO}$ & $\begin{array}{c}r=0,33 \\
p=0,004\end{array}$ & $\begin{array}{c}r=0,27 \\
p=0,018\end{array}$ & $\begin{array}{c}r=0,35 \\
p=0,003\end{array}$ & $\begin{array}{c}r=0,29 \\
p=0,0012\end{array}$ & НД & $\begin{array}{c}r=0,27 \\
p=0,018\end{array}$ & НД \\
\hline $\mathrm{HF}$ & $r=0,25 p=0,02$ & нд & $\begin{array}{c}r=0,3 \\
p=0,0042\end{array}$ & $\begin{array}{c}r=0,38 \\
p=0,0002\end{array}$ & $\begin{array}{c}r=0,28 \\
p=0,016\end{array}$ & $r=0,25 p=0,02$ & $\begin{array}{c}r=0,27 \\
p=0,018\end{array}$ \\
\hline
\end{tabular}

Основным маркером, определяющим артериальную ригидность, является скорость распространения пульсовой волны (СРПВ), которую можно исследовать различными способами. В настоящее время эталонным методом считают измерение на каротидно-феморальном сегменте при помощи аппланационной тонометрии. При этом производится запись сфигмограмм с сонной и бедренной артерий с одновременной регистрацией ЭКГ. В настоящее время появилась возможность измерения данного показателя в течение суток при проведении CMAД аппаратом BPLab, но метод определения по двум точкам неприменим для суточного мониторирования. Вместе с тем, как указано в литературе, для оценки СРПВ можно использовать время распространения отраженной от бифуркации аорты пульсовой волны [13].

В группе женщин с остеопорозом СРПВ средняя за сутки была выше на 2\% по сравнению с контролем. В предыдущих публикациях нами было показано, что у женщин с наличием остеопороза СРПВ, измеренная на кародидно-феморальном сегменте, при помощи аппланационной тонометрии также демонстрировала статистически значимое превышение по сравнению с группой, не имеющей остеопоротических переломов и снижения МПКТ [14]. Помимо увеличения СРПВ, нами проанализированы и другие показатели ригидности артерий. Так установлено, что в группе женщин с остеопорозом индекс ригидности артерий (ASI), был на 1,6\% выше по сравнению с контролем. В исследованиях показана прямая связь между индексом ригидности артерий и риском развития ИБС [15]. В 2006 году введено понятие амбулаторного индекса жесткости артерий (AASI), определяемого расчетным путем по уровню САД и ДАД [16]. В первой группе данный показатель был выше на 25,9\%.

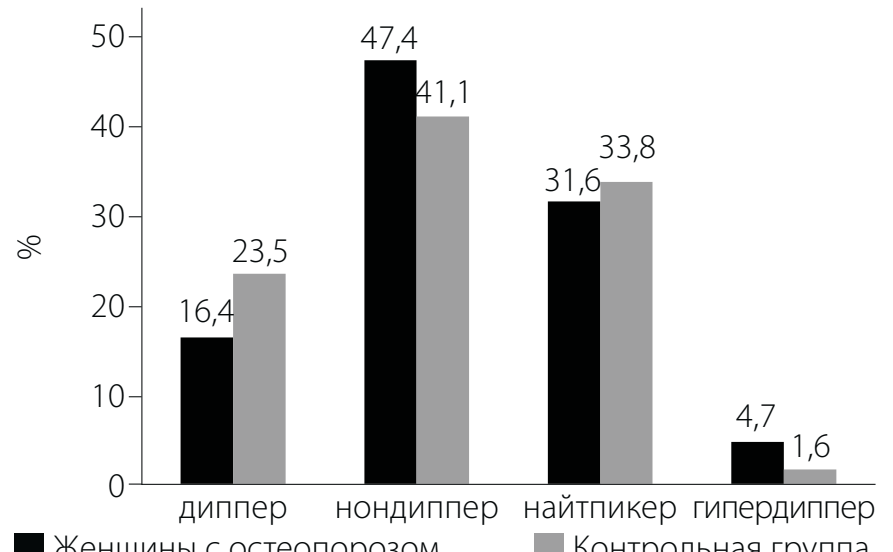

Женщины с остеопорозом

Контрольная группа

Рис. 1. Профили центрального систолического аортального давления.

\section{Дополнительные результаты исследования}

Для установления взаимосвязи между параметрами центрального давления и артериальной ригидности и наличием в анамнезе остеопоротических переломов (учитывался как качественный признак) проведен корреляционный анализ с использование ү-корреляции, а также оценивались корреляционные взаимосвязи между количеством остеопортических переломов в анамнезе, десятилетним абсолютным риском остеопоротических переломов, перелома шейки бедра и параметрами центрального давления и жесткости артерий. Поскольку в данном случае взаимосвязь определялась между количественными величинами, была использована ранговая корреляция Спирмена. Данные представлены в таблице 3.

Гамма-корреляцией выявлена положительная связь между уровнями САД, ДАД, средним АД, индексом аугментации, приведенным к ЧСС, в аорте, амбулаторным индексом ригидности и наличием у пациенток остеопоротических переломов в анамнезе. В результате ранговой корреляции установлена прямая связь между уровнями САД, ДАД, средним АД, средним индексом аугментации, приведенным к ЧСС, в аорте, амбулаторным индексом ригидности и абсолютным десятилетним риском остеопоротических переломов, а также количеством остеопоротических переломов в анамнезе. Показатель абсолютного десятилетнего риска перелома шейки бедра был положительно связан с уровнями САД, средним АД, средним индексом аугментации, приведенным к ЧСС, в аорте, средним пульсовым давлением, индексом ригидности и амбулаторным индексом ригидности.

Изучение степени ночного снижения центрального САД у пациенток с постменопаузальным остеопорозом вы-

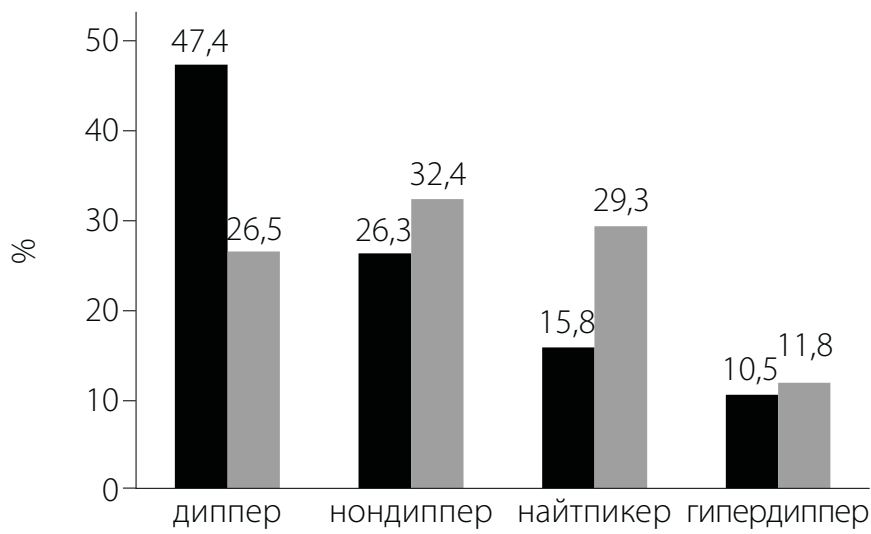

Женщины с остеопорозом

Рис. 2. Профили центрального диастолического аортального давления. 
явило, что лишь у 16,4\% женщин в данной группе регистрировался профиль диппер (с достаточным снижением САД в ночное время) против группы контроля, в которой данный профиль встречался у 23,5\% пациенток. Относительно патологических профилей следует отметить, что у женщин с остеопорозом тип гипердиппер (с избыточным снижением САД) встречался чаще (Рис. 1).

Профили диастолического давления демонстрировали обратную тенденцию. Так, у женщин с остеопорозом нормальный суточный профиль диппер ДАДао встречался чаще, чем в группе контроля 47,4\% против 26,5\% (Рис. 2). Среди патологических профилей в контрольной группе преобладал тип найтпикер (повышение ДАДао ночью) в 1,85 раза чаще.

Тип гипердиппер встречался с одинаковой частотой, тип нондиппер преобладал в группе сравнения.

\section{Нежелательные явления}

Не фиксировались в ходе исследования.

\section{ОБСУЖДЕНИЕ}

\section{Резюме основного результата исследования}

В результате проведенного нами исследования впервые установлено, что у женщин с постменопаузальным остеопорозом при суточном мониторировании отмечаются более высокие показатели центрального давления в восходящей части аорты и параметры артериальной ригидности.

\section{Обсуждение основного результата исследования}

Известно, что показатели центрального давления в той или иной степени зависят от жесткости артерий. Так, выявленное нами статистически значимое повышение давления амплификации в аорте может свидетельствовать о том, что увеличение систолического давления в аорте ассоциировано с феноменом аугментации давления, что может быть связано с более высокой ригидностью артерий в группе пациенток с остеопорозом. Физиологический смысл амплификации пульсовой волны состоит в препятствии угасанию ее на периферии, т. е. происходит «подпитывание» энергией нисходящей пульсовой волны, в результате чего САД на периферии увеличивается [17]. С другой стороны, отраженные волны, образующиеся в зонах высокого импеданса, распространяясь в восходящем направлении, суммируются, возвращаются к устью аорты и накладываются на центральную волну, ее диастолическую часть. Однако при ригидных артериях скорость отраженных пульсовых волн возрастает и, возвращаясь к центру, они накладываются на систолическую часть центральной волны, что приводит к повышению САД в аорте.
Это в свою очередь увеличивает постнагрузку на левый желудочек.

Маркером жесткости артерий является такой показатель как СРПВ. В нашем исследовании выявлено увеличение данного показателя в группе женщин с остеопорозом. В работе Скрипниковой И.А. и соавт., 2015 г. продемонстрировано, что у женщин с низкой костной массой по сравнению с контрольной группой СРПВ, измеренная методом аппланационной тонометрии, также была выше [10].

Кроме того, в ходе нашего исследования были установлены взаимосвязи между некоторыми параметрами центрального давления, артериальной ригидности и наличием переломов, их количеством и риском их возникновения, что может косвенно указывать на наличие возможной патогенетической связи сердечно-сосудистых заболеваний и остеопороза, которая широко обсуждается в последнее время.

\section{Ограничения исследования}

Истинная достоверность полученных статистически значимых результатов может быть не высокой в связи с небольшой выборкой пациенток. Для подтверждения выявленных взаимосвязей требуется проведение исследований по схожему протоколу с большим количеством участниц.

\section{ЗАКЛЮЧЕНИЕ}

В заключение следует отметить, что у женщин с остеопорозом выявлено повышение среднесуточных показателей САД, ДАД и среднего давления в аорте. Установлено, что в данной группе пациентов увеличены основные показатели жесткости артерий: СРПВ, ASI, AASI, PPA. Выявлена прямая корреляционная связь между параметрами центрального давления, артериальной ригидности и наличием в анамнезе переломов, их количеством, а также показателями абсолютного десятилетнего риска остеопоротических переломов и перелома шейки бедра. У пациенток с остеопорозом чаще выявляются патологические профили систолического давления в аорте, преобладающим из которых был тип гипердиппер.

\section{ДОПОЛНИТЕЛЬНАЯ ИНФОРМАЦИЯ.}

Источник финансирования. ФГБОУ ВО Читинская государственная медицинская академия

Конфликт интересов. Авторы декларируют отсутствие явных и потенциальных конфликтов интересов, связанных с публикацией настоящей статьи.

Благодарности. Авторы выражают благодарность рентгенологам НУз Дорожная клиническая больница на ст. Чита-2 за проведение денситометрии.

\section{СПИСОК ЛИТЕРАТУРЫ | REFERENCES}

1. Davies JI, Struthers AD. Pulse wave analysis and pulse wave velocity: a critical review of their strengths and weaknesses. J Hypertens. 2003;21(3):463-472. doi: 10.1097/01.hjh.0000052468.40108.43.

2. Лопатин Ю.М., Илюхин О.В., Илюхина М.В., Иваненко В.В. Эластичность артерий и скорость пульсовой волны у больных с хронической сердечной недостаточностью различной этиологии // Сердечная недостаточность. - 2004. - Т. 4. - №5. - С. 130-131. [Lopatin YM, Ilyuhin OV Ilyuhina MV, Ivanenko WV. Elastichnost' arteriy i skorost' pul'sovoy volny u bol'nykh s khronicheskoy serdechnoy nedostatochnost'yu razlichnoy etiologii. Zhurnal serdechnaia nedostatochnost' 2004;4(5):130-131 (In Russ.)].

3. Недогода С.В., Лопатин Ю.М., Чаляби Т.А., и др. Изменение скорости распространения пульсовой волны при артериальной гипертензии // Южно-Российский медицинский журнал. 2002. - №3. - C. 39-43. [Nedogoda SV, Lopatin YM, Chalyabi TA, et al. Izmenenie skorosti rasprostraneniya pul'sovoy volny pri arterial'noy 
gipertenzii. Yuzhno-Rossiyskiy meditsinskiy zhurnal. 2002;(3):39-43. (In Russ.)].

4. Орлова Я.А., Агеев Ф.Т. Жесткость артерий как интегральный показатель сердечнососудистого риска: физиология, методы оценки и медикаментозной коррекции // Сердце. - 2006. - Т. 5. - №2. - С. 65-68. [Orlova YA, Ageev FT. Zhestkost' arteriy kak integral'nyy pokazatel' serdechnososudistogo riska: fiziologiya, metody otsenki i medikamentoznoy korrektsii. Serdce. 2006;5(2):65-69 (In Russ.)].

5. Милягин В.А., Филичкин Д.Е., Шпынев К.В., и др. Контурный анализ центральной и периферической пульсовых волн у здоровых людей и больных артериальной гипертонией. // Артериальная гипертензия. - 2009. - Т. 15. - №1. - C. 78-85. [Milyagin VA, Filichkin DE, Shpynev KV, et al. Countour analysis of central and peripheral pulse waves in healthy subjects and in hypertensive. Arterial'naia gipertenziia. 2009;15(1):78-85. (In Russ.)]

6. Аксенова Т.А., Горбунов В.В., Пархоменко Ю.В, Царенок С.Ю. Суточное мониторирование центрального аортального давления и показателей артериальной ригидности при сочетании гипертонической болезни с хронической обструктивной болезнью легких. // Забайкальский медицинский вестник. - 2012. - №. 2. - C. 9-16. [Aksenova TA, Gorbunov W, Parkhomenko YV, Tsarenok SY. Daily monitoring of the central aortic pressure and arterial stiffness in combination with hypertension and chronic obstructive pulmonary disease. Zabajkal'skij medicinskij vestnik. 2012;(2):9-16 (In Russ.)].

7. Чазова И.Е., Сметник В.П., Балан В.Е., и др. Ведение женщин с сердечно-сосудистым риском в пери- и постменопаузе: консенсус российских кардиологов и гинекологов. // Системные гипертензии. 2008. - №. 3. - C. 26-39. [Chazova IE, Smetnik VP, Balan VE, et al. Vedenie zhenshchin s serdechno-sosudistym riskom v peri- i postmenopauze: konsensus rossiyskikh kardiologov i ginekologov. Sistemnye gipertenzii. 2008;(3):26-39 (In Russ.)].

8. Vogt MT, Valentin RS, Forrest KYZ, et al. Bone Mineral Density and Aortic Calcification: The Study of Osteoporotic Fractures. J Am Geriatr Soc. 1997;45(2):140-145. doi: 10.1111/j.1532-5415.1997.tb04498.x.

9. Hak AE, Pols HAP, van Hemert AM, et al. Progression of Aortic Calcification Is Associated With Metacarpal Bone Loss During Menopause: A Population-Based Longitudinal Study. Arterioscler Thromb Vasc Biol. 2000;20(8):1926-1931. doi: 10.1161/01.atv.20.8.1926.
10. Скрипникова И.А., Алиханова Н.А., Ткачева О.Н., И др. Минеральная плотность кости и состояние сосудистой стенки в зависимости от статуса репликативного клеточного старения у женщин в постменопаузальном периоде // Остеопороз и остеопатии. 2015. - T. 18. - №3. - C. 13-17. [Skripnikova IA, Alikhanova NA, Tkacheva ON, et al. Mineral'naya plotnost' kosti i sostoyanie sosudistoy stenki v zavisimosti ot statusa replikativnogo kletochnogo stareniya u zhenshchin v postmenopauzal'nom periode. Osteoporosis and Bone Diseases. 2015;18(3):13-17. (In Russ.)] doi: 10.14341/osteo2015313-17.

11. Rogoza A, Kuznetsov. Central aortic blood pressure and augmentation index: comparison between Vasotens\&reg; and SphygmoCor\&reg; technology. Research Reports in Clinical Cardiology. 2012:27. doi: 10.2147/rrcc.s30994.

12. Алексеева ЛИ, Баранова ИА, Белова КЮ, и др. Клинические рекомендации по профилактике и ведению больных с остеопорозом. - Ярославль: Литера, 2012. [Alekseeva LI, Baranova IA, Belova KY, et al. Klinicheskie rekomendacii po profilaktike i vedeniyu bol'nyh s osteoporozom. Yaroslavl: Litera; 2012 (In Russ.)].

13. London G, Guerin A, Pannier B, et al. Increased systolic pressure in chronic uremia. Role of arterial wave reflections. Hypertension. 1992;20(1):10-19. doi: 10.1161/01.hyp.20.1.10.

14. ЦЦаренок С.Ю., Горбунов В.В. Контурный анализ центральной пульсовой волны и эластические свойства артерий у женщин с ИБС и высоким риском остеопоротических переломов. // Забайкальский медицинский вестник. - 2014. - №. 4. - С. 159-163. [Tsarenok SY, Gorbunov W Contour analysis of the central pulse wave and elastic properties of arteries in women with CHD and high risk of osteoporotic fractures. Zabajkal'skij medicinskij vestnik. 2014;(4):159-163 (In Russ.)].

15. Cardio Vision Featuring The Arterial Stiffness Index (ASI) Selected Papers, Letters, and Pertinent Information. Cardio Vision is Distributed by: IMDP [электронный ресурс] режим доступа: http://www.imdp. com/media/pdf/imdpcvbrochure.pdf (24/07/2012)

16. Dolan E, Thijs L, Li Y, et al. Ambulatory Arterial Stiffness Index as a Predictor of Cardiovascular Mortality in the Dublin Outcome Study. Hypertension. 2006;47(3):365-370. doi: 10.1161/01.HYP.0000200699.74641.c5.

17. Nichols WW, O'Rourke MF, Vlachopoulos C. McDonald's Blood Flow in Arteries, Sixth Edition: Theoretical, Experimental and Clinical Principles. London: CRC Press; 2011.

\section{ИНФОРМАЦИЯ ОБ АВТОРАХ [AUTHORS INFO]}

Царенок Светлана Юрьевна, K.M.н. [Svetlana Yu. Tsarenok, MD, PhD]; адрес: Россия. 672000, Чита, улица Лермонтова 10 , кв. 10 [address: 10 Lermontov street, fl. 10, 672000, Chita, Russian]; телефон +79144659883; ORCID: http://orcid.org/0000-0002-7065-5737; eLibrary SPIN: 6086-0889; e-mail: sveta-tsarenok@ yandex.ru

Горбунов Владимир Владимирович, д.м.н., професcop [Vladimir V. Gorbunov, MD, PhD, Proffessor], телефoн +7914470-29-33, ORCID: http://orcid.org/00000002-3811-2943; eLibrary SPIN: 5628-2334 e-mail: gorbunovvv2008@mail.ru

Аксенова Татьяна Александровна, Д.м.Н., доцент [Tatiana A. Aksenova, MD, PhD], телефон +79144681651; ORCID: http://orcid.org/0000-0003-4957-5908; eLibrary SPIN: 3275-2360, e-mail: tatianaks@mail.ru

\section{ЦИТИРОВАТЬ:}

Царенок С.Ю., Горбунов В.В., Аксенова Т.А. Центральное аортальное давление и артериальная ригидность при суточном мониторировании у женщин с постменопаузальным остеопорозом // Остеопороз и остеопатии. — 2017. — Т. 20. — №2. — C.46-51. doi: 10.14341/osteo9368

\section{TO CITE THIS ARTICLE:}

Tsarenok SY, Gorbunov VV, Aksenova TA. The central aortic blood pressure and arterial stiffness during the daily monitoring procedure in postmenopausal women with osteoporosis. Osteoporosis and bone diseases. 2017;20(2):46-51. doi: 10.14341/osteo9368 